\title{
VALUATIONS OF SKEW QUANTUM POLYNOMIALS
}

\author{
Cristian Arturo Chaparro Acosta \\ crachaparroac@unal.edu.co \\ Seminario de Álgebra Constructiva - $\mathrm{SAC}^{2}$. \\ Departamento de Matemáticas. \\ Universidad Nacional de Colombia. \\ Sede Bogotá.
}

\begin{abstract}
In this paper we extend some results obtained by Artamonov and Sabitov for quantum polynomials to skew quantum polynomials and quasi-commutative bijective skew PBW extensions. Moreover, we find a counterexample to the conjecture proposed in [6].
\end{abstract}

Keywords: Skew $P B W$ extensions, skew quantum polynomials, Ore domains, valuations, completions.

\section{Contents}

1 Introduction 2

1.1 Valuations . . . . . . . . . . . . . . . . 2

1.2 Valuations with values on $\Gamma \cup\{\infty\} \ldots \ldots \ldots$

1.3 Quantum polynomials ................ 3

2 Completions of quantum polynomials 4

3 Skew $P B W$ extensions 6

3.1 Skew quantum polynomials . . . . . . . . . . . . . 9

3.2 Some properties . . . . . . . . . . . . . . . . 10

3.3 Valuations of skew quantum polynomials. . . . . . . . . 16

3.4 Valuations of skew $P B W$ extension. . . . . . . . . . . 16

$\begin{array}{ll}\text { References } & 16\end{array}$ 


\section{Introduction}

This section is divided into three subsections, we recall the definition of $\Gamma$-valuation, valuation and quantum polynomials. We review some fundamental properties of valuations and valuations of quantum polynomials (see [4] and [6]).

\section{$1.1 \quad$ Valuations}

Let $D$ be a division ring, $D^{*}$ the multiplicative group and $\Gamma$ is a totally ordered group (with additive notation and not necessarily commutative).

Definition 1.1. A function $\nu: D^{*} \rightarrow \Gamma$ is a $\Gamma$-valuation of $D^{*}$ if:

i) $\nu$ is surjective,

ii) $\nu(a b)=\nu(a)+\nu(b)$,

iii) $\nu(a+b) \geq \min \{\nu(a), \nu(b)\}$.

Proposition 1.2. [14, 9] If $\nu$ is a $\Gamma$-valuation of $D^{*}$, then:

1) If $\nu(a) \neq \nu(b)$, then $\nu(a+b)=\min \{\nu(a), \nu(b)\}$.

2) $\Lambda_{\nu}:=\{a \in D ; a=0$ or $\nu(a) \geq 0\}$ is a subring of $D$.

3) The group of units $\mathcal{U}_{\nu}:=\left\{a \in D^{*} ; \nu(a)=0\right\}$ is a subgroup of $D^{*}$.

4) $\mathcal{W}_{\nu}:=\{a \in D, a=0$ or $\nu(a)>0\}$ is a completely prime ideal of $\Lambda_{\nu}$ and $\mathcal{W}_{\nu}=\Lambda_{\nu}-\mathcal{U}_{\nu}$

5) $\Lambda_{\nu}$ is a local ring with unique maximal ideal $\mathcal{W}_{\nu}$.

\subsection{Valuations with values on $\Gamma \cup\{\infty\}$}

Proposition 1.3. Let $\Gamma$ be a totally ordered group with additive notation ordere. Then $\Gamma \cup\{\infty\}$ is an ordered additive monoid such that

$$
x+\infty:=\infty=: \infty+x, \text { for all } \Gamma \cup\{\infty\},
$$

and $\infty>x$ for all $x \in \Gamma$.

Definition 1.4 ([8]). Let $R$ be a ring. By a valuation on $R$ with values in a totally ordered group $\Gamma$, the value group, we shall understand a function $\nu$ on $R$ with values in $\Gamma \cup\{\infty\}$ subject to the conditions:

i) $\nu(a) \in \Gamma \cup\{\infty\}$ and $\nu$ assumes at least two values,

ii) $\nu(a b)=\nu(a)+\nu(b)$, 
iii) $\nu(a+b) \geq \min \{\nu(a), \nu(b)\}$.

Proposition 1.5. [8, 9] If $\nu$ is a valuation of $R$, then:

1) $\operatorname{ker} \nu:=\{a \in R ; \nu(a)=\infty\}$ is an ideal of $R$.

2) If $\nu(a+b)>\min \{\nu(a), \nu(b)\}$, then $\nu(a)=\nu(b)$.

3) $\Lambda_{\nu}:=\{a \in R ; \nu(a) \geq 0\}$ is a subring of $R$.

4) The group of units $\mathcal{U}_{\nu}:=\left\{a \in R^{*} ; \nu(a)=0\right\}$ is a subgroup of $R^{*}$.

5) $\mathcal{W}_{\nu}:=\{a \in R, \nu(a)>0\}$ is an ideal of $\Lambda_{\nu}$.

6) ker $\nu$ is a completely prime ideal of $R$ and $R /$ ker $\nu$ is an integral domain.

Proposition 1.6 ([8]). If $\nu$ is a $\Gamma$-valuation of $D$. Then $\Gamma$ is abelian, if and only if $\nu(a)=0$ for all $a \in\left[D^{*}, D^{*}\right]$.

\subsection{Quantum polynomials}

Let $D$ be a division ring with a fixed set $\alpha_{1}, \alpha_{2}, \ldots, \alpha_{n}, n \geq 2$, of automorphimsms. Also, we have $q_{i j}$ in $D^{*}$ for $i, j=1,2, \ldots, n$ fix elements, satisfying the relations :

$$
\begin{gathered}
q_{i i}=q_{i j} q_{j i}=\boldsymbol{q}_{i j r} \boldsymbol{q}_{j r i} \boldsymbol{q}_{r i j}=1 \\
\alpha_{i}\left(\alpha_{j}(d)\right)=q_{i j} \alpha_{j}\left(\alpha_{i}(d)\right) q_{j i},
\end{gathered}
$$

where $\boldsymbol{q}_{i j r}=q_{i j} \alpha_{j}\left(q_{i r}\right)$ and $d \in D$. We set $\boldsymbol{q}=\left(q_{i j}\right) \in \mathscr{M}(n, D)$ and $\alpha=\left(\alpha_{1}, \alpha_{2}, \ldots, \alpha_{n}\right)$.

Definition 1.7. The elements $q_{i j}$ of the matrix $\boldsymbol{q}$ are called system of multiparameters.

Definition 1.8 (Quantum polynomial ring). Denote by

$$
\mathcal{O}_{q}:=D_{\boldsymbol{q}, \alpha}\left[x_{1}^{ \pm 1}, x_{2}^{ \pm 1}, \ldots, x_{r}^{ \pm 1}, x_{r+1}, \ldots, x_{n}\right],
$$

the associative ring generated by $D$ and by elements $x_{1}^{ \pm 1}, x_{2}^{ \pm 1}, \ldots, x_{r}^{ \pm 1}$, $x_{r+1}, \ldots, x_{n}$ subject to the defining relations

$$
\begin{gathered}
x_{i} x_{i}^{-1}=x_{i}^{-1} x_{i}=1,1 \leq i \leq r, \\
x_{i} d=\alpha_{i}(d) x_{i}, d \in D, i=1,2, \ldots, n, \\
x_{i} x_{j}=q_{i j} x_{j} x_{i}, i, j=1,2, \ldots, n .
\end{gathered}
$$


Definition 1.9. Let $N$ be the subgroup in the multiplicative group $D^{*}$ of the ring $D$ generated by the derived subgroup $\left[D^{*}, D^{*}\right]$ and by the set of all elements of the form $z^{-1} \sigma_{i}(z)$ where $z \in R^{*}$ and $i=1, \ldots, n . \Lambda:=$ $D_{q, \alpha}\left[x_{1}, x_{2}, \ldots, \ldots, x_{n}\right]$ is a general (generic) quantum polynomials ring if the images of all multiparameters $q_{i j}, 1 \leq i<j \leq n$, are independent in the multiplicative Abelian group $D^{*} / N$.

The ring $\mathcal{O}_{\boldsymbol{q}}$ is a left and right Noetherian domain, it satisfies Ore Condition and it has a division ring of fractions $F:=D_{q}\left(x_{1}, \ldots, x_{n}\right)$. We consider $\nu: F^{*} \rightarrow \Gamma$ a $\Gamma$-valuation with $\nu\left(D^{*}\right)=0$.

Theorem 1.10 ([6]). A valuation of a quantum division ring $D$, is Abelian in the sense that the group $\Gamma$ is Abelian.

Definition 1.11 ([4], [6]). Let $\nu_{1}: D^{*} \rightarrow \Gamma_{1}$ and $\nu_{2}: D^{*} \rightarrow \Gamma_{2}$ be two valuations. Set $\nu_{1} \geq \nu_{2}$ if there exists an epimorphism of ordered groups $\tau: \Gamma_{1} \rightarrow \Gamma_{2}$ such that $\tau \nu_{1}=\nu_{2}$. It means that the diagram

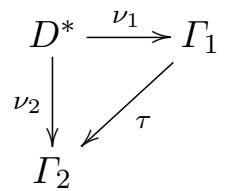

is commutative.

Definition 1.12 ([4], [6]). A valuation $\nu$ has a maximal rank if $\tau$ is an isomorphism in the previous definition.

Theorem $1.13([4])$. A valuation $\nu: F^{*} \rightarrow \Gamma$ of a general quantum division ring $\mathcal{O}_{q}$ is has maximal rank if only if $\Gamma \cong \mathbb{Z}^{n}$.

\section{Completions of quantum polynomials}

In this section $\nu: F^{*} \rightarrow \mathbb{Z}^{n}$ is a maximal $\mathbb{Z}^{n}$-valuation.

Definition $2.1([6])$. Let $\mathscr{F}$ be the set of all maps $f: \mathbb{Z}^{n} \rightarrow k$ and the zero element such that supp $f:=\left\{m \in \mathbb{Z}^{n} ; f(m) \neq 0\right\}$ is Artinian with respect to the lexicographic order on $\mathbb{Z}^{n}$.

Theorem 2.2. $\mathscr{F}$ is a division ring containing $F$.

Proof. See [3] Theorem 3.4 and 3.7.

Expand the valuation $\nu$ to $f \in \mathscr{F}$ in the following way. If $f \in \mathscr{F}$ then $\nu(f)$ the least element from supp $f$.

Definition $2.3([6])$. The division ring $\mathscr{F}$ is called a completion of $F$ with respect to $\nu$. 
Remark 2.4. If $\mathcal{O}:=\{f \in \mathscr{F} ; \nu(f) \geq 0\}$ and $\mathbf{m}:=\{f \in \mathscr{F} ; \nu(f)>0\}$, then $\mathcal{O}$ is a subring in $\mathscr{F}$ and $\mathbf{m}$ is an ideal in $\mathcal{O}$. Moreover, $\mathcal{O} / \mathbf{m} \cong k$.

Let $\mathbb{R}^{n}$ be a vector space of all rows $\left(r_{1}, \ldots, r_{n}\right), r_{i} \in \mathbb{R}$, of a length $n$ and $\mathbb{R}^{n}$ is equipped with the lexicographic order.

Theorem 2.5 ([10]). Let $\leq_{\mathbb{Z}^{n}}$ be a totally order in the additive group $\mathbb{Z}^{n}$. Then there exists order preserving group embedding $\mathbb{Z}^{n} \rightarrow \mathbb{R}^{n}$.

Definition 2.6. [6] A totally order $\leq_{\mathbb{Z}^{n}}$ is essentially lexicographic if it belongs to the orbit of the standard embedding of $\mathbb{Z}^{n}$ in to $\mathbb{R}^{n}$ under the action of the group $G L(n, \mathbb{Z})$. i.e., if $a, b \in \mathbb{Z}^{n}, a \leq \mathbb{Z}^{n} b$ if only if $a A \leq b A$ for some fixed $A$ in $G L(n, \mathbb{Z})$ and $\leq$ the lexicographic order.

Conjecture $2.7([6])$. A valuation $\nu$ is associated to an essentially lexicographic order on $\mathbb{Z}^{n}$ if and only if $\cap_{i>1} \boldsymbol{m}^{i}=0$.

In the study of this conjecture we obtain the following results partial:

Proposition 2.8. If $\nu: R \rightarrow \Gamma \cup\{\infty\}$ is a valuation of a ring $R$ and $\Gamma$ is a Archimedean group with $\mathcal{W}_{\nu}:=\{a \in R, \nu(a)>0\}$, $\inf \left\{\nu\left(\mathcal{W}_{\nu}\right)\right\} \neq 0$ and $\bigcap_{i \geq 1} \mathcal{W}_{\nu}^{i}:=I$, then $\nu(I)=\infty$.

Proof. Let $A_{i}:=\nu\left(\mathcal{W}_{\nu}^{i}\right)$ and $\lambda_{i}:=\inf \left\{A_{i}\right\}$ be, then $\lambda_{1}<\lambda_{2}<\ldots<\lambda_{i}$ and $i \lambda_{1} \leq \lambda_{i}$, indeed: (by induction over $i$ ) as $\inf \left\{\nu\left(\mathcal{W}_{\nu}\right)\right\} \neq 0$ then $0<\lambda_{1} \leq \nu(a)$ for all $a \in \mathcal{W}_{\nu}$, hence $\lambda_{1}<2 \lambda_{1} \leq \nu(a b)$ for all $a, b \in \mathcal{W}_{\nu}$, therefore $2 \lambda_{1} \leq \lambda_{2}$, suppose that $\lambda_{i-1}<\lambda_{i}$ and $i \lambda_{1} \leq \lambda_{i}$, then $i \lambda_{1}<$ $(i+1) \lambda_{1} \leq \lambda_{i}+\lambda_{1} \leq \nu(a)+\nu(b)=\nu(a b)$ for all $a \in \mathcal{W}_{\nu}^{i}$ and $b \in \mathcal{W}_{\nu}$, then, $\lambda_{i}<\lambda_{i+1}$ and $(i+1) \lambda_{1} \leq \lambda_{i+1}$.

Now, suppose there exists $b \in I$ such that $\nu(b)=\lambda<\infty$, so $\lambda_{1}<\lambda$ and as $\Gamma$ is Archimedean there exists an integer $m$ such that $m \lambda_{1}>\lambda$, therefore $\lambda \notin A_{m}$, hence $b \notin \mathcal{W}_{\nu}^{m}$, contradicting that $b \in I$.

Corollary 2.9. If $\nu: D \rightarrow \Gamma \cup\{\infty\}$ is a valuation of a division ring $D$ and $\Gamma$ is a Archimedean group with $\inf \left\{\nu\left(\mathcal{W}_{\nu}\right)\right\} \neq 0$, then $\bigcap_{i \geq 1} \mathcal{W}_{\nu}^{i}=0$.

Proof. $0=\nu(1)=\nu\left(a a^{-1}\right)=\nu(a)+\nu\left(a^{-1}\right)$ for all $a \in D^{*}$, then $\nu(a)<\infty$ for all $a \in D^{*}$, therefore $\nu(a)=\infty$ if only if $a=0$.

Remark 2.10. In the Proposition 2.8 the condition $\inf \left\{\nu\left(\mathcal{W}_{\nu}\right)\right\} \neq 0$ can be replaced by $\inf \left\{\nu\left(\mathcal{W}_{\nu}^{i}\right)\right\} \neq 0$ for any $i>0$ in $\mathbb{N}$.

Example 2.11. If we take lexicographic order on $\mathbb{Z}^{2}$ the order does not have intersection property: consider $A:=\left\{(x, y) \in \mathbb{Z}^{2} ;(0,0)<(x, y)\right\}$ and $n A:=\sum_{i=1}^{n} A$ with $n>0$, then $n A=\left\{(x, y) \in \mathbb{Z}^{2} ;(0, n) \leq(x, y)\right\}$. By induction over n: If $n=2$, then $2 A=A \backslash\{(0,1)\}$, indeed: as $\min \{A\}=(0,1)$ then $(0,2) \leq(x, y)$ with $(x, y) \in 2 A$, thus $2 A \subseteq A \backslash\{(0,1)\}$. Now, if $(x, y)$ 
in $2 A$, then $(x, y-1) \in A$, because $x>0$ or $x=0$ and $y \geq 2$.

Suppose that $n A=(n-1) A \backslash\{(0, n-1)\}$, as $\min \{n A\}=(0, n)$ then $(0, n+1) \leq(x, y)$ with $(x, y) \in(n+1) A$, thus $(n+1) A \subseteq n A \backslash\{(0, n)\}$. Now, if $(x, y)$ in $(n+1) A$, then $(x, y-1) \in n A$, because $x>0$ or $x=0$ and $y \geq n+1$. Consequently $(n+1) A=\left\{(x, y) \in \mathbb{Z}^{2} ;(0, n+1) \leq(x, y)\right\}$.

Hence, as $(1,0) \in n A$ for every $n \geq 1$ since $(0, n)<(1,0)$, then $(1,0) \in$ $\cap_{n>0} n A$.

It follows a counterexample to the conjecture, since a lexicographic order is essentially lexicographic.

\section{Skew $P B W$ extensions}

In this section we recall the definition and some basic properties of skew PBW (Poincaré-Birkhoff-Witt) extensions, introduced in [11]. Some ringtheoretic and homological properties of these class of noncommutative rings have been studied in [12].

Definition 3.1. Let $R$ and $A$ be rings. We say that $A$ is a skew $P B W$ extension of $R$ (also called a $\sigma-P B W$ extension of $R$ ) if the following conditions hold:

(i) $R \subseteq A$.

(ii) There exists finitely many elements $x_{1}, \ldots, x_{n} \in A$ such $A$ is a left $R$-free module with basis

$$
\operatorname{Mon}(A):=\left\{x^{u}=x_{1}^{u_{1}} \cdots x_{n}^{u_{n}} \mid u=\left(u_{1}, \ldots, u_{n}\right) \in \mathbb{N}^{n}\right\}
$$

In this case it also says that $A$ is a left polynomial ring over $R$ with respect to $\left\{x_{1}, \ldots, x_{n}\right\}$ and $\operatorname{Mon}(A)$ is the set of standard monomials of $A$. Moreover, $x_{1}^{0} \cdots x_{n}^{0}:=1 \in \operatorname{Mon}(A)$.

(iii) For every $1 \leq i \leq n$ and $r \in R-\{0\}$ there exists $c_{i, r} \in R-\{0\}$ such that

$$
x_{i} r-c_{i, r} x_{i} \in R .
$$

(iv) For every $1 \leq i, j \leq n$ there exists $c_{i, j} \in R-\{0\}$ such that

$$
x_{j} x_{i}-c_{i, j} x_{i} x_{j} \in R+R x_{1}+\cdots+R x_{n} .
$$

Under these conditions we will write $A:=\sigma(R)\left\langle x_{1}, \ldots, x_{n}\right\rangle$. 
Proposition 3.2. Let $A$ be a skew $P B W$ extension of $R$. Then, for every $1 \leq i \leq n$, there exists an injective ring endomorphism $\sigma_{i}: R \rightarrow R$ and $a$ $\sigma_{i}$-derivation $\delta_{i}: R \rightarrow R$ such that

$$
x_{i} r=\sigma_{i}(r) x_{i}+\delta_{i}(r),
$$

for each $r \in R$.

Proof. See [11], Proposition 3.

The previous proposition gives the notation and the alternative name given for the skew $P B W$ extensions.

Definition 3.3. Let $A$ be a skew $P B W$ extension.

(a) A is quasi-commutative if the conditions (iii) and (iv) in Definition 3.1 are replaced by

(iii') For every $1 \leq i \leq n$ and $r \in R-\{0\}$ there exists $c_{i, r} \in R-\{0\}$ such that

$$
x_{i} r=c_{i, r} x_{i} .
$$

(iv') For every $1 \leq i, j \leq n$ there exists $c_{i, j} \in R-\{0\}$ such that

$$
x_{j} x_{i}=c_{i, j} x_{i} x_{j}
$$

(b) $A$ is bijective if $\sigma_{i}$ is bijective for every $1 \leq i \leq n$ and $c_{i, j}$ is invertible for any $1 \leq i<j \leq n$.

Definition 3.4. Let $A$ be a skew $P B W$ extension of $R$ with endomorphisms $\sigma_{i}, 1 \leq i \leq n$, as in Proposition 3.2.

(i) For $u=\left(u_{1}, \ldots, u_{n}\right) \in \mathbb{N}^{n}, \sigma^{u}:=\sigma_{1}^{u_{1}} \cdots \sigma_{n}^{u_{n}},|u|:=u_{1}+\cdots+u_{n}$. If $v=\left(v_{1}, \ldots, v_{n}\right) \in \mathbb{N}^{n}$, then $u+v:=\left(u_{1}+v_{1}, \ldots, u_{n}+v_{n}\right)$.

(ii) For $X=x^{u} \in \operatorname{Mon}(A), \exp (X):=u$ and $\operatorname{deg}(X):=|u|$.

(iii) If $f=c_{1} X_{1}+\cdots+c_{t} X_{t}$, with $X_{i} \in M o n(A)$ and $c_{i} \in R-\{0\}$, then $\operatorname{deg}(f):=\max \left\{\operatorname{deg}\left(X_{i}\right)\right\}_{i=1}^{t}$.

Theorem 3.5. Let $A$ be a left polynomial ring over $R$ w.r.t. $\left\{x_{1}, \ldots, x_{n}\right\}$. $A$ is a skew PBW extension of $R$ if and only if the following conditions hold:

(a) For every $x^{u} \in \operatorname{Mon}(A)$ and every $0 \neq r \in R$ there exist unique elements $r_{u}:=\sigma^{u}(r) \in R-\{0\}$ and $p_{u, r} \in A$ such that

$$
x^{u} r=r_{u} x^{u}+p_{u, r},
$$

where $p_{u, r}=0$ or $\operatorname{deg}\left(p_{u, r}\right)<|u|$ if $p_{u, r} \neq 0$. Moreover, if $r$ is left invertible, then $r_{u}$ is left invertible. 
(b) For every $x^{u}, x^{v} \in \operatorname{Mon}(A)$ there exist unique elements $c_{u, v} \in R$ and $p_{u, v} \in A$ such that

$$
x^{u} x^{v}=c_{u, v} x^{u+v}+p_{u, v},
$$

where $c_{u, v}$ is left invertible, $p_{u, v}=0$ or $\operatorname{deg}\left(p_{u, v}\right)<|u+v|$ if $p_{u, v} \neq 0$.

Proof. See [11], Theorem 7.

Proposition 3.6. Let $A$ be a skew $P B W$ extension of a ring $R$. If $R$ is a domain, then $A$ is a domain.

Proof. See [12].

The next theorem characterizes the quasi-commutative skew $P B W$ extensions.

Theorem 3.7. Let $A$ be a quasi-commutative skew $P B W$ extension of a ring $R$. Then,

(i) A is isomorphic to an iterated skew polynomial ring of endomorphism type, i.e.,

$$
A \cong R\left[z_{1} ; \theta_{1}\right] \cdots\left[z_{n} ; \theta_{n}\right] .
$$

(ii) If $A$ is bijective, then each endomorphism $\theta_{i}$ is bijective, $1 \leq i \leq n$.

Proof. See [12].

Corollary 3.8. Let $A$ be a bijective and quasi-commutative skew PBW extension of a ring $R$. If $R$ is a left Ore domain, then $A$ is a left Ore domain.

Proof. By Theorem 3.7, $A$ is isomorphic to an iterated skew polynomial ring of automorphism type over a left Ore domain $R$.

Theorem 3.9. Let $A$ be an arbitrary skew $P B W$ extension of $R$. Then, $A$ is a filtered ring with filtration given by

$$
F_{m}:= \begin{cases}R & \text { if } m=0 \\ \{f \in A \mid \operatorname{deg}(f) \leq m\} & \text { if } m \geq 1\end{cases}
$$

and the corresponding graded ring $G r(A)$ is a quasi-commutative skew $P B W$ extension of $R$. Moreover, if $A$ is bijective, then $\operatorname{Gr}(A)$ is a quasi-commutative bijective skew $P B W$ extension of $R$.

Proof. See [12].

Theorem 3.10 (Hilbert Basis Theorem). Let $A$ be a bijective skew PBW extension of $R$. If $R$ is a left (right) Noetherian ring then $A$ is also a left (right) Noetherian ring.

Proof. See [12]. 


\subsection{Skew quantum polynomials}

In this subsection we recall the definition and some basic properties of skew quantum polynomials ring over $R$, introduced in [12]. We mention some results generalized for valuations of skew quantum polynomials and bijective and quasi-commutative skew $P B W$ extension.

Definition 3.11. Let $R$ be a ring with matrix of parameters $q:=\left[q_{i j}\right] \in$ $M_{n}(R), n \geq 2$, such that $q_{i i}=1=q_{i j} q_{j i}=q_{j i} q_{i j}$ for each $1 \leq i, j \leq n$ and suppose also that is given a system $\sigma_{1}, \ldots, \sigma_{n}$ of automorphisms of $R$. The skew quantum polynomials ring over $R$, denoted by

$$
R_{\boldsymbol{q}, \sigma}\left[x_{1}^{ \pm 1}, \ldots, x_{r}^{ \pm 1}, x_{r+1}, \ldots, x_{n}\right]
$$

is defined whit the following conditions:

i) $R \subseteq R_{\boldsymbol{q}, \sigma}\left[x_{1}^{ \pm 1}, \ldots, x_{r}^{ \pm 1}, x_{r+1}, \ldots, x_{n}\right]$,

ii) $R_{q, \sigma}\left[x_{1}^{ \pm 1}, \ldots, x_{r}^{ \pm 1}, x_{r+1}, \ldots, x_{n}\right]$ is a free left $R$-module with basis $\left\{x^{u} ; x^{u}=\right.$ $x_{1}^{u_{1}} \cdots x_{n}^{u_{n}}, u_{i} \in \mathbb{Z}, 1 \leq i \leq r$ and $u_{i} \in \mathbb{N}$ for $\left.r+1 \leq i \leq n\right\}$,

iii) The $x_{1}, \ldots, x_{n}$ elements satisfy the defining relations

$$
\begin{gathered}
x_{i} x_{i}^{-1}=1=x_{i}^{-1} x_{i}, 1 \leq i \leq r, \\
x_{i} x_{j}=q_{j i} x_{j} x_{i} 1 \leq i, j \leq n, \\
x_{i} r=\sigma_{i}(r) x_{i}, r \in R y 1 \leq i \leq n .
\end{gathered}
$$

When all automorphisms are trivial, we write $R_{\boldsymbol{q}}\left[x_{1}^{ \pm 1}, \ldots, x_{r}^{ \pm 1}, x_{r+1}, \ldots\right.$, $x_{n}$ ] and this ring is called the ring of quantum polynomials over $R$. If $R=K$ is a field, then $K_{q, \sigma}\left[x_{1}^{ \pm 1}, \ldots, x_{r}^{ \pm 1}, x_{r+1}, \ldots, x_{n}\right]$ is the algebra of skew quantum polynomials. For trivial automorphisms we get the algebra of quantum polynomials simply.

If $r=n, R_{q, \sigma}\left[x_{1}^{ \pm 1}, \ldots, x_{n}^{ \pm 1}\right]$ is called the $n$-multiparametric skew quantum torus over $R$, when all automorphisms are trivial, is called the $n$-multiparametric quantum torus over $R$. If $r=0, R_{q, \sigma}\left[x_{1}, \ldots, x_{n}\right]$ is called the $n$ multiparametric skew quantum space over $R$, when all automorphisms are trivial is called $n$-multiparametric quantum space over $R$.

The algebra of quantum polynomials can be defined as a quasi-commutative bijective skew $P B W$ extension of the $r$-multiparameter quantum torus, or also, as a localization of a quasi-commutative bijective skew $P B W$ extension. 
Theorem 3.12. $R_{\boldsymbol{q}, \sigma}\left[x_{1}, \ldots, x_{n}\right] \cong R\left[z_{1} ; \theta_{1}\right] \cdots\left[z_{n} ; \theta_{n}\right]$, where
i) $\theta_{1}=\sigma_{1}$
ii) $\theta_{i}: R\left[z_{1} ; \theta_{1}\right] \cdots\left[z_{i-1} ; \theta_{i-1}\right] \rightarrow R\left[z_{1} ; \theta_{1}\right] \cdots\left[z_{i-1} ; \theta_{i-1}\right]$
iii) $\theta_{i}\left(z_{i}\right)=q_{i j} z_{i}, 1 \leq i<j \leq n, \theta_{i}(r)=\sigma_{i}(r)$ for $r \in R$.

In particular, $R_{q}\left[x_{1}, \ldots, x_{n}\right] \cong R\left[z_{1}\right] \cdots\left[z_{n} ; \theta_{n}\right]$.

Proof. See [12].

Theorem 3.13. $R_{\boldsymbol{q}, \sigma}\left[x_{1}^{ \pm 1}, \ldots, x_{r}^{ \pm 1}, x_{r+1} \ldots, x_{n}\right]$ is a ring of fractions of $B:=R_{q, \sigma}\left[x_{1}, \ldots, x_{n}\right]$ with respect to the multiplicative subset

$$
S=\left\{r x^{u} ; r \in R^{*}, x^{u} \in \operatorname{Mon}\left\{x_{1}, \ldots, x_{r}\right\}\right\},
$$

i.e,

$$
R_{\boldsymbol{q}, \sigma}\left[x_{1}^{ \pm 1}, \ldots, x_{r}^{ \pm 1}, x_{r+1} \ldots, x_{n}\right] \cong S^{-1} B .
$$

Proof. See [12].

Remark 3.14. Let $Q_{\boldsymbol{q}, \sigma}^{r, n}(R):=R_{\boldsymbol{q}, \sigma}\left[x_{1}^{ \pm 1}, \ldots, x_{r}^{ \pm 1}, x_{r+1}, \ldots, x_{n}\right]$ and $R$ be a left (right) Noetherian ring, then $Q_{q, \sigma}^{r, n}(R)$ is left (right) Noetherian by Theorem 3.10. Moreover, if $R$ is a domain, then $Q_{q, \sigma}^{r, n}(R)$ is also a domain by Theorem 3.6. Thus, if $R$ is a left (right) Noetherian domain, then $Q_{\boldsymbol{q}, \sigma}^{r, n}(R)$ is a left (right) Ore domain.

Thus, $Q_{q, \sigma}^{r, n}(R)$ has a total division ring of fractions

$$
Q\left(Q_{q, \sigma}^{r, n}(R)\right) \cong Q(A):=\sigma(R)\left(x_{1}, \ldots, x_{n}\right),
$$

where $\sigma(R)\left(x_{1}, \ldots, x_{n}\right)$ denotes the rational fractions of $A:=\sigma(R)\left\langle x_{1}, \ldots, x_{n}\right\rangle$.

\subsection{Some properties}

Definition 3.15. Let $N$ be the subgroup in the multiplicative group $R^{*}$ of the ring $R$ generated by the derived subgroup $\left[R^{*}, R^{*}\right]$ and by the set of all elements of the form $z^{-1} \sigma_{i}(z)$ where $z \in R^{*}$ and $i=1, \ldots, n$.

Remark 3.16. $N$ is a normal subgroup in $R^{*}$.

Definition 3.17. If the images of $q_{i j}$ with $1 \leq i<j \leq n$ are independent in the multiplicative Abelian group $\bar{R}=R^{*} / N$ then, $R_{\boldsymbol{q}, \sigma}\left[x_{1}^{ \pm 1}, \ldots, x_{r}^{ \pm 1}, x_{r+1}\right.$, $\left.\ldots, x_{n}\right]$ is a generic skew quantum polynomials ring.

Remark 3.18. If $\mathrm{n}=2$ in $R_{q, \sigma}\left[x_{1}^{ \pm 1}, \ldots, x_{r}^{ \pm 1}, x_{r+1}, \ldots, x_{n}\right]$, of the previous definition $q=q_{12}$ is not a root of unity.

Proposition 3.19. For each $a \in R^{*}$ and $\sigma$ endomorphism over $R, \sigma^{k}(a)=$ an with $k \in \mathbb{N}$ and $n \in N$. 
Proof.

$$
\begin{aligned}
\sigma^{k}(a) & =a\left(a^{-1} \sigma(a)\right)\left((\sigma(a))^{-1} \sigma^{2}(a)\right) \ldots\left(\left(\sigma^{k-1}(a)\right)^{-1} \sigma^{k}(a)\right) \\
& =a n, \text { with } n \in N .
\end{aligned}
$$

Proposition 3.20. If $u, v \in \mathbb{Z}^{r} \times \mathbb{N}^{n-r}$ and $\lambda, \mu \in R^{*}$, then

(1) $x_{i} x^{u}=\left(\prod_{j=1}^{n} q_{j i}^{u_{j}}\right) n_{u} \cdot x^{u} x_{i}$, for some $n_{u} \in N$ and for any $1 \leq i \leq n$.

(2) $\left(x^{u}\right)\left(x^{v}\right)=\left(\prod_{i<j} q_{j i}^{u_{j} v_{i}}\right) n_{u+v} \cdot x^{u+v}$, with $n_{u+v} \in N$.

(3) $\left(\lambda x^{u}\right)\left(\mu x^{v}\right)=\lambda \mu\left(\prod_{i<j} q_{j i}^{u_{j} v_{i}}\right) n^{\prime} \cdot x^{u+v}$, with $n^{\prime} \in N$.

Proof. Applying the Proposition 3.19 and note that $x_{i} x_{j}^{-1}=q_{j i}^{-1} x_{j}^{-1} x_{i}$ with $1 \leq j \leq r$.

Proposition 3.21. Let $f:=\sum_{u \in \mathbb{Z}} \lambda_{u} x^{u}$ be in $R_{q, \sigma}\left[x_{1}^{ \pm 1}, \ldots, x_{r}^{ \pm 1}, x_{r+1}, \ldots, x_{n}\right]$ and $x_{i}$ with $1 \leq i \leq r$.

(1) If $\lambda_{u} \in R$, then

$$
x_{i} f x_{i}^{-1}=\sum_{u \in \mathbb{Z}^{n}} \sigma_{i}\left(\lambda_{u}\right) \lambda_{u}^{\prime} x^{u},
$$

where $\lambda_{u}^{\prime}:=\left(\prod_{j=1}^{n} q_{j i}^{u_{j}}\right) n_{u} \in R^{*}$.

(2) If $\lambda_{u} \in R^{*}$, then

$$
x_{i} f x_{i}^{-1}=\sum_{u \in \mathbb{Z}^{n}} \lambda_{u}^{\prime} x^{u},
$$

where $\lambda_{u}^{\prime} \in R^{*}$.

Proof. (1) Note that $N \subseteq R^{*}$ and

$$
\begin{aligned}
x_{i} f x_{i}^{-1} & =\sum \sigma_{i}\left(\lambda_{u}\right) x_{i} x^{u} x^{-i} \\
& =\sum_{u \in \mathbb{Z}^{n}} \sigma_{i}\left(\lambda_{u}\right)\left(\prod_{j=1}^{n} q_{j i}^{u_{j}}\right) n_{u} x^{u},
\end{aligned}
$$

where $n_{u} \in N$.

(2) By item (1), $\sigma_{i}\left(\lambda_{u}\right) \lambda_{u}^{\prime} \in R^{*}$ 
Remark 3.22. If $Q\left(Q_{\boldsymbol{q}, \sigma}^{r, n}(R)\right)$ exists and $G$ denotes the multiplicative subgroup in $Q\left(Q_{q, \sigma}^{r, n}(R)\right)^{*}$ generated by $R^{*}$ and $x_{1}, \ldots, x_{n}$. Then $R^{*} \triangleleft G$ and $G / R^{*}$ is a free abelian group with the base $x_{1} R^{*}, \ldots, x_{n} R^{*}$.

Proposition 3.23. Let $R$ be a left Ore domain and $\sigma$ automorphisms over $R$, then $\sigma$ can be extended to $Q(R)$ and is also an automorphism.

Proof. By universal property we have the following commutative diagram:

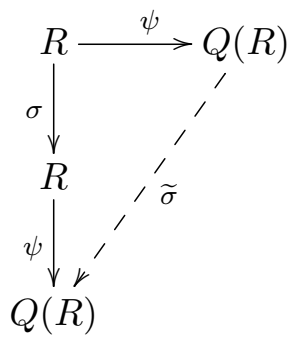

where $\psi, \sigma$ are injective and $\widetilde{\sigma}\left(\frac{a}{b}\right)=\frac{\sigma(a)}{\sigma(b)}$ for $a, b \neq 0 \in R$. Therefore, $\psi \circ \sigma$ is injective and so is $\widetilde{\sigma}$.

If $\frac{a}{b} \in Q(R)$, then $\frac{a}{b}=\psi(b)^{-1} \psi(a)=\psi\left(\sigma\left(b_{0}\right)\right)^{-1} \psi\left(\sigma\left(a_{0}\right)\right)$ for $a_{0}, b_{0} \neq$ $0 \in R$, consequently,

$$
\begin{aligned}
\frac{a}{b} & =\psi\left(\sigma\left(b_{0}\right)\right)^{-1} \psi\left(\sigma\left(a_{0}\right)\right) \\
& =\widetilde{\sigma}\left(\psi\left(b_{0}\right)\right)^{-1} \widetilde{\sigma}\left(\psi\left(a_{0}\right)\right) \\
& =\widetilde{\sigma}\left(\psi\left(b_{0}\right)^{-1} \psi\left(a_{0}\right)\right) \\
& =\widetilde{\sigma}\left(\frac{a_{0}}{b_{0}}\right) .
\end{aligned}
$$

Theorem 3.24. Let $R$ be a left Ore domain and $S=R-\{0\}$, then

$$
S^{-1}\left(R_{\boldsymbol{q}, \sigma}\left[x_{1}, \ldots, x_{n}\right]\right) \cong Q(R)_{\tilde{\boldsymbol{q}}, \widetilde{\sigma}}\left[x_{1}, \ldots, x_{n}\right],
$$

where $\widetilde{\boldsymbol{q}}=\left(\frac{q_{i j}}{1}\right) \in \mathscr{M}(n, Q(R))$.

Proof. By Theorem $3.12 R_{\boldsymbol{q}, \sigma}\left[x_{1}, \ldots, x_{n}\right] \cong R\left[z_{1} ; \theta_{1}\right] \cdots\left[z_{n} ; \theta_{n}\right]$, with each $\theta_{i}$ bijective. Thus, if $S=R-\{0\}$ then

$$
\begin{aligned}
S^{-1}\left(R_{q, \sigma}\left[x_{1}, \ldots, x_{n}\right]\right) & \cong S^{-1}\left(R\left[z_{1} ; \theta_{1}\right] \cdots\left[z_{n} ; \theta_{n}\right]\right) \\
& \cong S^{-1}(R)\left[z_{1} ; \widetilde{\theta_{1}}\right] \cdots\left[z_{n} ; \widetilde{\theta_{n}}\right] \\
& =Q(R)\left[z_{1} ; \widetilde{\theta_{1}}\right] \cdots\left[z_{n} ; \widetilde{\theta_{n}}\right]
\end{aligned}
$$


where

$$
\begin{aligned}
\widetilde{\theta_{1}}: Q(R) & \rightarrow Q(R) \\
\frac{a}{b} & \mapsto \widetilde{\theta_{1}}\left(\frac{a}{b}\right)=\frac{\theta_{1}(a)}{\theta_{1}(b)}=\frac{\sigma_{1}(a)}{\sigma_{1}(b)}=\widetilde{\sigma_{1}}\left(\frac{a}{b}\right)
\end{aligned}
$$

and

$$
\widetilde{\theta_{i}}: Q(R)\left[z_{1} ; \widetilde{\theta_{1}}\right] \cdots\left[z_{i-1} ; \widetilde{\theta_{i-1}}\right] \rightarrow Q(R)\left[z_{1} ; \widetilde{\theta_{1}}\right] \cdots\left[z_{i-1} ; \widetilde{\theta_{i-1}}\right]
$$

with

$$
\widetilde{\theta_{i}}\left(\frac{a}{b}\right)=\widetilde{\sigma}_{i}\left(\frac{a}{b}\right) \text { y } \widetilde{\theta_{j}}\left(z_{i}\right)=\frac{q_{i j}}{1} z_{i}
$$

Therefore,

$$
S^{-1}\left(R_{\boldsymbol{q}, \sigma}\left[x_{1}, \ldots, x_{n}\right]\right) \cong Q(R)_{\tilde{\boldsymbol{q}}, \widetilde{\sigma}}\left[x_{1}, \ldots, x_{n}\right],
$$

where $\widetilde{\boldsymbol{q}}=\left(\frac{q_{i j}}{1}\right) \in \mathscr{M}(n, Q(R))$.

Proposition 3.25. Let $R$ be a left Ore domain, there exists

$$
\phi: R_{\boldsymbol{q}, \sigma}\left[x_{1}^{ \pm 1}, \ldots, x_{n}^{ \pm 1}\right] \rightarrow Q(R)_{\tilde{\boldsymbol{q}}, \widetilde{\sigma}}\left[x_{1}^{ \pm 1}, \ldots, x_{n}^{ \pm 1}\right]
$$

an injective ring homomorphism.

Proof. Let $B_{R}:=R_{q, \sigma}\left[x_{1}, \ldots, x_{n}\right]$ and $B_{Q(R)}:=Q(R)_{\tilde{q}, \tilde{\sigma}}\left[x_{1}, \ldots, x_{n}\right]$ be, by Theorem $3.13 R_{q, \sigma}\left[x_{1}^{ \pm 1}, \ldots, x_{n}^{ \pm 1}\right] \cong S_{1}^{-1} B_{R}$ with $S_{1}=\left\{r x^{u} ; r \in R^{*}\right.$, $\left.x^{u} \in \operatorname{Mon}\left\{x_{1}, \ldots, x_{n}\right\}\right\}$, and $Q(R)_{\tilde{q}, \tilde{\sigma}}\left[x_{1}^{ \pm 1}, \ldots, x_{n}^{ \pm 1}\right] \cong S_{1^{\prime}}^{-1} B_{Q(R)}$ with $S_{1^{\prime}}=$ $\left\{r x^{u} ; r \in Q(R)^{*}, x^{u} \in \operatorname{Mon}\left\{x_{1}, \ldots, x_{n}\right\}\right\}$.

Now, consider the following diagram of ring homomorphisms:

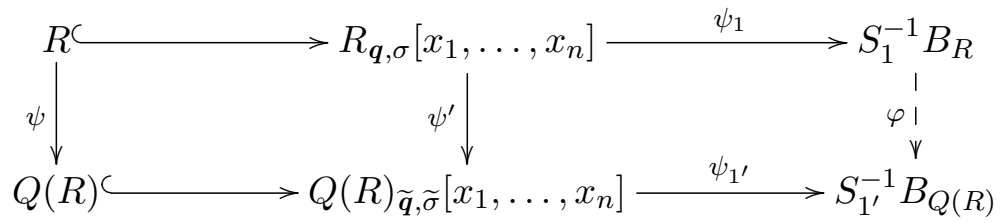

where $\psi$ is the injection for the localization of $R$ to the total ring fractions $Q(R), \psi^{\prime}$ the injection determined by the isomorphism of Theorem 3.24 where $\psi^{\prime}\left(a x^{u}\right)=\frac{a}{1} x^{u}$, and $\psi_{1}, \psi_{1^{\prime}}$ injections determined by the localizations for $B_{R}$ and $B_{Q(R)}$ respectively.

As $\psi^{\prime}\left(S_{1}\right) \subseteq S_{1^{\prime}}$, then $\psi_{1^{\prime}}\left(\psi^{\prime}\left(S_{1}\right)\right) \subseteq \psi_{1^{\prime}}\left(S_{1^{\prime}}\right) \subseteq\left(S_{1^{\prime}}^{-1} B_{Q(R)}\right)^{*}$, therefore, by universal property there exists $\varphi$. If $f=\sum a_{u} x^{u} \in R_{\boldsymbol{q}, \sigma}\left[x_{1}, \ldots, x_{n}\right]$ and $r x^{v} \in S_{1}$ then, 


$$
\begin{aligned}
\varphi\left(\frac{f}{r x^{v}}\right) & =\varphi\left(\frac{\sum a_{u} x^{u}}{r x^{v}}\right) \\
& =\psi_{1^{\prime}}\left(\psi^{\prime}\left(r x^{v}\right)\right)^{-1} \psi_{1^{\prime}}\left(\psi^{\prime}\left(\sum a_{u} x^{u}\right)\right) \\
& =\psi_{1^{\prime}}\left(\frac{r}{1} x^{v}\right)^{-1} \psi_{1^{\prime}}\left(\sum \frac{a_{u}}{1} x^{u}\right) \\
& =\frac{\frac{1}{1}}{\frac{r}{1} x^{v}} \frac{\sum \frac{a_{u}}{1} x^{u}}{\frac{1}{1}} \\
& =\frac{\sum \frac{a_{u}}{1} x^{u}}{\frac{r}{1} x^{v}} \\
& =\frac{\psi^{\prime}(f)}{\psi^{\prime}\left(r x^{v}\right)} .
\end{aligned}
$$

Also, $\varphi$ is injective by $\psi^{\prime}$ and $\psi_{1^{\prime}}$ are injective.

Need the following result for the subsequent theorem:

Proposition 3.26. Let $R$ be $a$ ring and $S \subset R$ a multiplicative subset. If $Q:=S^{-1} R$ exists, then any finite set $\left\{q_{1}, \ldots, q_{n}\right\}$ of elements of $Q$ posses a common denominator, i.e., there exists $r_{1}, \ldots, r_{n} \in R$ and $s \in S$ such that $q_{i}=\frac{r_{i}}{s}, 1 \leq i \leq n$.

Proof. See [13], Lemma 2.1.8.

Theorem 3.27. Let $R$ be a left Ore domain, then $Q\left(Q_{q, \sigma}^{n, n}(R)\right) \cong Q\left(Q_{\widetilde{\boldsymbol{q}}, \widetilde{\sigma}}^{n, n}(Q(R))\right.$.

Proof. With the notation of the proof in the Proposition 3.25 consider the following diagram of ring homomorphisms

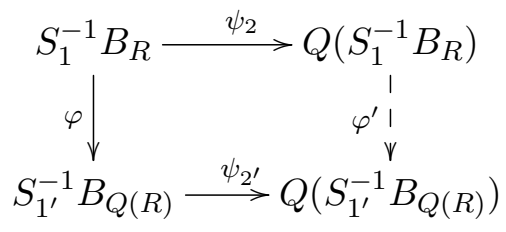

where $\psi_{2}, \psi_{2^{\prime}}$ are injections determined by the localizations of $S_{1}^{-1} B_{R}$ and $S_{1^{\prime}}^{-1} B_{Q(R)}$ respectively and $\varphi$ the injection of the Proposition 3.25.

By Remark 3.14, $S_{1}^{-1} B_{R}$ and $S_{1^{\prime}}^{-1} B_{Q(R)}$ are domain, now, if $\frac{p_{1}}{q_{1}}, \frac{p_{2}}{q_{2}} \in$ $S_{1}^{-1} B_{R}$ with $\frac{p_{1}}{q_{1}} \neq 0$, then $p_{1} \neq 0$ and there exist $f_{1} \neq 0$ and $f_{2} \in$ $B_{R}$ such that $f_{1} p_{1}=f_{2} p_{2}$. Then, $\frac{f_{1} q_{1}}{1} \frac{p_{1}}{q_{1}}=\frac{f_{1} p_{1}}{1}=\frac{f_{2} q_{2}}{1}=\frac{f_{2} q_{2}}{1} \frac{p_{2}}{q_{2}} \neq 0$, therefore $S_{1}^{-1} B_{R}$ is a Ore domain, similarly it has to $S_{1^{\prime}}^{-1} B_{Q(R)}$. Thus, if $S_{2}=S_{1}^{-1} B_{R}-\{0\}$ and $S_{2^{\prime}}=S_{1^{\prime}}^{-1} B_{Q(R)}-\{0\}$ as $\varphi\left(S_{2}\right) \subseteq S_{2^{\prime}}$, then 
$\psi_{2^{\prime}}\left(\varphi\left(S_{2}\right)\right) \subseteq \psi_{2^{\prime}}\left(S_{2^{\prime}}\right) \subseteq\left(Q\left(S_{1^{\prime}}^{-1} B_{Q(R)}\right)\right)^{*}$, hence, by universal property there exists $\varphi^{\prime}$ injective ring homomorphism.

Note that if $f, g \in B_{R}$ and $a x^{u}, b x^{b} \in S_{1}$, then

$$
\frac{\frac{f}{a x^{u}}}{\frac{g}{b x^{v}}}=\left(\frac{g}{b x^{v}}\right)^{-1} \frac{f}{a x^{u}}=\frac{b x^{v}}{g} \frac{f}{a x^{u}}=\frac{f^{\prime}}{g^{\prime}}
$$

and

$$
\frac{f^{\prime}}{g^{\prime}}=\frac{1}{g^{\prime}} \frac{f^{\prime}}{1}=\left(\frac{g^{\prime}}{1}\right)^{-1} \frac{f^{\prime}}{1}=\frac{\frac{f^{\prime}}{1}}{\frac{g^{\prime}}{1}},
$$

where $f^{\prime}, g^{\prime} \in B_{R}$ by Remark 3.14 with $r=0$. Similarly is obtained for $Q\left(S_{1^{\prime}}^{-1} B_{Q}(R)\right)$.

Therefore,

$$
\begin{aligned}
\varphi^{\prime}\left(\frac{f}{g}\right) & =\psi_{2^{\prime}}\left(\varphi\left(\frac{g}{1}\right)\right)^{-1} \psi_{2^{\prime}}\left(\varphi\left(\frac{f}{1}\right)\right) \\
& =\psi_{2^{\prime}}\left(\frac{\psi^{\prime}(g)}{\frac{1}{1}}\right)^{-1} \psi_{2^{\prime}}\left(\frac{\psi^{\prime}(f)}{\frac{1}{1}}\right) \\
& =\frac{\frac{1}{1}}{\psi^{\prime}(g)} \frac{\psi^{\prime}(f)}{\frac{1}{1}} \\
& =\frac{\psi^{\prime}(f)}{\psi^{\prime}(g)} .
\end{aligned}
$$

Now, if $f, 0 \neq g \in S_{1^{\prime}}^{\prime} B_{Q(R)}$, applying Theorem 3.26 must be

$$
\begin{aligned}
\frac{f}{g} & =\frac{\sum \frac{a_{u}}{b_{u}} x^{u}}{\sum \frac{c_{v}}{d_{v}} x^{v}}=\frac{\frac{1}{s} \sum \frac{a_{u}^{\prime}}{1} x^{u}}{\frac{1}{s^{\prime}} \sum \frac{c_{v}^{\prime}}{1} x^{v}}=\left(\sum \frac{c_{v}^{\prime}}{1} x^{v}\right)^{-1}\left(\frac{1}{s^{\prime}}\right)^{-1} \frac{1}{s} \sum \frac{a_{u}^{\prime}}{1} x^{u} \\
& =\left(\sum \frac{c_{v}^{\prime}}{1} x^{v}\right)^{-1}\left(\frac{s^{\prime}}{1} \frac{1}{s}\right) \sum \frac{a_{u}^{\prime}}{1} x^{u}=\left(\sum \frac{c_{v}^{\prime}}{1} x^{v}\right)^{-1}\left(\frac{r^{\prime}}{r}\right) \sum \frac{a_{u}^{\prime}}{1} x^{u} \\
& =\left(\sum \frac{c_{v}^{\prime}}{1} x^{v}\right)^{-1}\left(\frac{1}{r} \frac{r^{\prime}}{1}\right) \sum \frac{a_{u}^{\prime}}{1} x^{u}=\left(\frac{r}{1} \sum \frac{c_{v}^{\prime}}{1} x^{v}\right)^{-1}\left(\frac{r^{\prime}}{1} \sum \frac{a_{u}^{\prime}}{1} x^{u}\right) \\
& =\left(\sum \frac{r c_{v}^{\prime}}{1} x^{v}\right)^{-1}\left(\sum \frac{r^{\prime} a_{u}^{\prime}}{1} x^{u}\right) \\
& =\frac{\sum \frac{r^{\prime} a_{u}^{\prime}}{1} x^{u}}{\sum \frac{r c_{v}^{\prime}}{1} x^{v}}=\frac{\psi^{\prime}\left(f^{\prime}\right)}{\psi^{\prime}\left(g^{\prime}\right)} \\
& =\varphi\left(\frac{f^{\prime}}{g^{\prime}}\right) .
\end{aligned}
$$

where $f^{\prime}=\sum\left(r^{\prime} a_{u}^{\prime}\right) x^{u}$ y $g^{\prime}=\sum\left(r c_{v}^{\prime}\right) x^{v}$, then $\varphi$ is surjective. Hence $Q\left(Q_{q, \sigma}^{n, n}(R)\right) \cong Q\left(Q_{\widetilde{\boldsymbol{q}}, \widetilde{\sigma}}^{n, n}(Q(R))\right)$. 


\subsection{Valuations of skew quantum polynomials.}

Theorem 3.28. Let $R$ be a left Ore domain and $\nu: Q\left(Q_{q, \sigma}^{n, n}(R)\right)^{*} \rightarrow \Gamma$ is a valuation with $\nu\left(Q(R)^{*}\right)=0$, then $\Gamma$ is Abelian.

Proof. $Q(R)$ is a division ring and $Q\left(Q_{\boldsymbol{q}, \sigma}^{n, n}(R)\right) \cong Q\left(Q_{\widetilde{\boldsymbol{q}}, \widetilde{\sigma}}^{n, n}(Q(R))\right)$, by Theorem 1.10. $\Gamma$ is Abelian.

Corollary 3.29. Let $R$ be a left Ore domain, $\nu: Q\left(Q_{q, \sigma}^{n, n}(R)\right)^{*} \rightarrow \Gamma$ a valuation with $\nu\left(Q(R)^{*}\right)=0$ and $Q_{\widetilde{\mathbf{q}}, \tilde{\sigma}}^{n, n}(Q(R))$ generic, then $\Gamma$ is Abelian.

Theorem 3.30. Let $R$ be a left Ore domain, a valuation $\nu: Q\left(Q_{q, \sigma}^{n, n}(R)\right)^{*} \rightarrow$ $\Gamma$ with $\nu\left(Q(R)^{*}\right)=0$ and $Q_{\widetilde{\boldsymbol{q}}, \tilde{\sigma}}^{n, n}(Q(R))$ generic. The valuation $\nu$ has maximal rank if only if $\Gamma \cong \mathbb{Z}^{n}$.

Proof. By Theorem 3.27. $Q\left(Q_{\boldsymbol{q}, \sigma}^{n, n}(R)\right) \cong Q\left(Q_{\widetilde{\boldsymbol{q}}, \widetilde{\sigma}}^{n, n}(Q(R))\right)$ with $Q(R)$ a division ring, by Theorem 1.13 the valuation $\nu$ has maximal rank if only if $\Gamma \cong \mathbb{Z}^{n}$.

\subsection{Valuations of skew $P B W$ extension.}

Theorem 3.31. Let $A=\sigma(R)\left\langle x_{1}, \ldots, x_{n}\right\rangle$ be a bijective and quasi-commutative skew $P B W$ extension of a ring $R$. If $R$ is a left Ore domain and $\nu: Q(A)^{*} \rightarrow$ $\Gamma$ a valuation with $\nu\left(Q(R)^{*}\right)=0$, then $\Gamma$ is Abelian

Proof. By Theorem $3.8 A$ is an Ore domain then, $Q(A)$ exists and is a division ring, by Remark 3.14. $Q(A) \cong Q\left(Q_{\boldsymbol{q}, \sigma}^{r, n}(R)\right.$ ) (in particular $r=0$ ) and by Theorem $3.28 \Gamma$ is abelian.

Corollary 3.32. Let $A$ be a bijective skew $P B W$ extension of a ring $R$. If $R$ is a left Ore domain and $\nu: Q(G r(A))^{*} \rightarrow \Gamma$ a valuation with $\nu\left(Q(R)^{*}\right)=0$, then $\Gamma$ is Abelian.

Proof. By Theorem $3.9 \operatorname{Gr}(A)$ is bijective and quasi-commutative.

\section{References}

[1] Artamonov V. A., Serre's Quantum Problem, Uspehi Mat. Nauk. 53 (1998), N 4, 3-76.

[2] Artamonov V. A., General quantum polynomials: irreducible modules and Morita-equivalence, Izv. RAN, Ser. Math. 63 (1999), N 5, 3-36.

[3] Artamonov V. A., Automorphisms of the skew field of rational quantum functions, Sbornik: Mathematics 191: 12 (2000).

[4] Artamonov V. A., Valuations on quantum fields, Commun. Algebra, 29 (2001), N 9. 
[5] Artamonov V. A., Actions of Hopf algebras on general quantum Mal'tsev power series and quantum planes. J. Math. Sci. (2006). 134, N 1. p. $1773-1798$.

[6] Artamonov V. A., Quantum polynomial, Advances in Algebra and Combinatorics, World Scientific, (2008).

[7] Cohn P. M., Algebraic Numbers and Algebraic Functions . Chapman \& Hall, $2-6$ boundary Row, London, 1991.

[8] Cohn P. M., Skew Fields Theory of General Division Rings, Cambridge University Press, 1995.

[9] Freddy Van Oystaeyen, Algebraic geometry for associative algebras. Marcel Dekker Inc. NY, (2000), 302 pp.

[10] Kokorin A. I. \& Kopytov V. M., Linearly ordered groups. Moscow: Nauka, (1972).

[11] Lezama O. \& Gallego C., Gröbner bases for ideals of sigma-PBW extensions, Communications in Algebra, 39 (1), 2011, 50-75.

[12] Lezama O. \& Reyes M., Some homological properties of skew PBW extensions, Communications in Algebra, Vol. 42, 2014, 1200-1230.

[13] McConnell J. \& Robson J., Non-commutative Noetherian Rings, Graduate Studies in Mathematics, AMS, 2001.

[14] Schilling O. F., Noncommutative Valuation, Bull. Amer. Math. Soc. Volume 51, Number 4 (1945), 229-324. 